\title{
Rib Fracture Stabilization Reduces Chest Wall Pain following Blunt Thoracic Trauma
}

\section{Subrato J. Deb*}

University of Oklahoma, Oklahoma City, USA

\begin{abstract}
Background: The optimal management of patients sustaining minor chest wall injury is controversial. Current trauma guidelines emphasize pain control and expectant management. Adjunctive measures to decrease pain would be beneficial. The objective of this study was to determine if displaced rib fracture fixation could lessen thoracic pain when conventional analgesia was not effective.
\end{abstract}

Methods: A retrospective analysis of 25 patients who underwent rib fracture stabilization over 44 months was undertaken. Only patients with Blunt Thoracic Trauma (BTT) and no other significant injuries were included. Numeric Pain Intensity (NPI) was used as a measure of the extent of thoracic pain. Patients with severe pain (NPI>7) despite alternative methods of analgesia were offered rib plating. NPI scores were measured at various time points and analyzed.

Results: A significant reduction in the level of perceived pain was noted among the cohort of patients who accepted rib fixation. A majority of patients ( $88 \%$ ) experienced reduction of chest wall pain within 48 hours of surgery. The mean NPI score was $8.9 \pm 1.1$ prior to fracture stabilization and reduced to $4.7 \pm 1.7(p<0.05)$ following surgery and further decreased to $2.9 \pm 1.3(p<0.05)$ at dismissal. At a median follow up of 4 months, recurrence of thoracic pain was not observed in any of the cohort and no complications specifically related to fracture plating was noted.

Conclusions: Displaced rib fracture reduction and stabilization appears to help reduce chest wall pain. This technology should be considered in the pain management algorithm for patients with severe pain from rib injury.

Keywords: Rib fracture; Blunt trauma; Thoracic pain; Stabilization

\section{Introduction}

Blunt Thoracic Trauma (BTT) encompasses a wide spectrum of varying pathologic conditions of the skeletal chest wall, pulmonary parenchyma and mediastinum [1-5]. Rib fractures are one of the most common injuries sustained following BTT and result in significant disability, largely due to pain [1]. Among chest trauma patients, up to $10 \%$ of all admissions have evidence of rib fracture, with mortality rates that can exceed $20 \%$ in some series [1-4]. Older patients and those with associated pulmonary injury experience the highest rates of death [1,3-5]. A consensus management strategy of patients sustaining skeletal chest wall injury remains undefined, largely due to the lack of randomized comparative data [3-5]. Rib fractures are often one component of a larger spectrum of thoracic trauma where the focus of care is toward organ function. To date, most of the available literature focuses upon those patients with severe chest wall injury where the treatment strategy is sustaining organ function and is directed by trauma society guidelines [4]. Patients with lesser degrees of injury to the thorax are treated with optimization of chest wall mechanics through chest physiotherapy and effective analgesia. Contemporary recommendations for pain management validate intravenous and epidural analgesia as the optimal modalities for control of thoracic pain $[3,4]$. The use of open reduction and internal fixation of broken ribs is debatable as these techniques are morbid and traditionally reserved to severely injured patients where the goal is to improve respiratory dynamics [4,5]. However, among BTT patients with isolated rib fracture and lesser concomitant injury, the treatment goal is alleviation of pain as noted previously [3]. A cohort of such patients, particularly those with displaced rib fractures, will continue to suffer severe pain, despite maximal medical therapy. In such patients, there may be a benefit to the application of rib fracture plating as a complimentary strategy for better pain control and decreased narcotic requirement [6-
8]. The objective of this paper is to determine if rib fracture plating can be beneficial in the pain management algorithm of patients sustaining BTT who have predominantly displaced rib fracture injury.

\section{Methods and Patients}

\section{Pain measurement}

The Numeric Pain Intensity (NPI) rating scale was recorded from patient charts as a quantitative measurement of the level of thoracic pain. This pain scale is validated by the National Institutes of Health Pain Consortium as a measure of individual pain intensity and as an indicator of the effectiveness of pain treatment. This scale ranges from 0 (no pain) to 10 (worst pain) and is illustrated in Table 1. Qualitative descriptions of pain made previously in this paper are based on the scale represented in Table 1. Pain intensity scores were abstracted from the available hospital records at various points in the patient's clinical course. Average daily NPI scores were recorded for each patient prior to surgery, at 24 and 48 hours following surgery, at dismissal and followup to simplify analysis. The NPI score the day prior to surgery was recorded as the baseline level of pain to which statistical comparisons were made.

*Corresponding author: Subrato J. Deb, University of Oklahoma, Oklahoma City, USA, Tel: +1 405-325-0311; E-mail: subrato-deb@ouhsc.edu

Received February 08, 2014; Accepted March 24, 2014; Published March 26 2014

Citation: Deb JS (2014) Rib Fracture Stabilization Reduces Chest Wall Pain following Blunt Thoracic Trauma. J Trauma Treat S2: 004. doi:10.4172/2167-1222. S2-004

Copyright: (c) 2014 Deb JS. This is an open-access article distributed under the terms of the Creative Commons Attribution License, which permits unrestricted use, distribution, and reproduction in any medium, provided the original author and source are credited. 


\begin{tabular}{|l|l|}
\hline $0=$ & No Pain \\
\hline $1-3=$ & Mild Pain (nagging, annoying, interfering little with ADLs) \\
\hline $4-6=$ & Moderate Pain (interferes significantly with ADLs) \\
\hline $7-10=$ & Severe Pain (disabling; unable to perform ADLs) \\
\hline
\end{tabular}

ADLs: Activities of Daily Living.

Table 1: Numeric Pain Intensity Rating Scale.

\section{Patients selection}

Between September 2009 and May 2013, 25 consecutive at a Level II trauma center with predominantly BTT underwent open reduction and internal fixation of displaced rib fractures. This cohort was selected from patients who had sustained blunt force trauma to the thorax and were admitted under the care of the trauma service. Patients were referred for chest wall stabilization by either the attending trauma surgeon or consulting pulmonologist if they had painful rib fractures that were not well managed with existing pain treatment methods. The timing of referral was at the discretion of the referring physician and varied between 1 and 28 days, with a median time interval of 3 days following injury. The decision to consider fracture plating was made in a multidisciplinary format involving the trauma service, anesthesiologist/ pain service, pulmonologist and thoracic surgeon. A unanimous consensus was required prior to proposing the procedure to the patients. Excluded were patients with major concomitant organ injury such as pulmonary contusion and flail chest injury. Patients who had displaced rib fractures without severe pain, those with nondisplaced rib fractures and those with distracting injuries causing pain were not offered rib fracture plating. Characterization of rib fracture anatomy was accomplished using chest radiography and multi-planar Computerized Axial Tomography (CT) prior to operative fixation.

\section{Patient characteristics}

The cohort consisted of 11 females and 14 males with a median age of 60 range 31 to 91 years. All patients sustained blunt force trauma. Sixteen patients (64\%) experienced a fall injury, eight patients had trauma related to a Motor Vehicle Crash (MVC) and one patient had a construction accident. In general, those patients sustaining fall injury were older, with a mean observed age of 62 years. Fractures in the patients who fell were mostly located at the point of impact injury. In contrast, patients who were involved in MVC were younger with a mean age of 49 years and the majority of fractures were located at the postero-lateral angle of the ribs. Eighteen patients had a hemothorax and 6 patients had pneumothorax as a result of rib fracture displacement and pleural violation. The number of rib fractures varied between 1 and 7 with a median of four. None of the patients had fractures of the first two and last two ribs. Concomitant injuries were relatively minor and as a group, these patients had a favorable projected probability of survival of $95.2 \pm 3.65$ based upon the TRISS calculation (Trauma ScoreInjury Severity Score). The TRISS is one indicator of the probability of survival following traumatic injury and is dependent upon the Injury Severity Score (ISS) and Revised Trauma Score (RTS). The ISS is an anatomic evaluation of organ injury following trauma and the RTS is a physiologic indicator of the patient's status upon presentation. Of this group of patients, sixteen patients (64\%) had left sided fractures and $36 \%$ had right sided fractures. All of the patients who underwent rib plaiting had a NPI score greater than or equal to 7 (severe pain), with 13 patients (52\%) experiencing maximum pain with a perceived level of 10 .

\section{Pain management prior to rib plating}

Pain management strategies prior to fracture plating among the cohort included thoracic epidural catheters in 9 (36\%). Patients were treated with Patient Controlled Analgesia (PCA) or oral narcotic medications if epidural catheters were not placed. PCA was used in $12(48 \%)$ patients and oral narcotic agents in the remaining 4. PCA infusions consisted of morphine in 9 of the 12 patients, with the remainder receiving hydromorphone. The formulation of the pain control plan for each patient was at the discretion of the trauma surgeon and the consulting anesthesiologist.

\section{Data collection and statistical analysis}

Data abstraction was conducted from hospital records, operative reports and clinic charts. Follow up was recorded as the most recent clinic visit from the time of hospital dismissal. Continuous variables are presented as mean \pm standard deviation. Parametric data such as the differences between levels of pain (NPI) at various time points was tested for significance with the students paired $t$ test. A probability value less than 0.05 was considered statistically significant. This study was reviewed by the Western Maryland Health System Clinical Research Oversight Team and IRB exemption was granted.

\section{Results}

\section{Surgical procedure}

Displaced rib fractures underwent open reduction and internal stabilization with commercially available minimally invasive rib plating systems (RibLoc, ACUTE Innovations, Hillsboro, OR and Biomet Micro fixation, Warsaw, IN). These systems allow the deployment of fracture stabilization plates through smaller access incisions, minimizing soft tissue injury. Fracture plates are available in different lengths and are malleable to accommodate individual chest wall contours. Access to the bony injury was made directly over the area of the fracture using muscle sparing techniques. Three dimensional CT reconstructions greatly facilitated the access to the fractured rib segments. Due to the simplicity of the utilized rib plating systems in this study, major thoracotomy was not required in any patient. The median number of ribs plated was 3 (range 1 to 5). In situations where comminuted bone fragments were encountered, the prosthetic plate was seated across all compromised bone and affixed to healthy bone at the ends of the rib injury to stabilize the moving elements. Eighteen patients (72\%) required concomitant tube thoracostomy for concomitant hemothorax. A representative chest radiograph of a patient who has undergone the procedure is demonstrated in Figure 1.

\section{Postoperative outcome}

There were no operative deaths or major complications related to the placement of the rib plating system among the cohort of patients. Two patients expired during the post-operative hospital course following rib stabilization from unrelated causes. One patient, who was 91 years old suffered a Cerebral Vascular Accident (CVA) on post-operative day 8 and expired on post-operative day 10 following the withdrawal of ventilator support. This patient had 3 displaced rib fractures repaired due to inadequate pain control with neuraxial analgesia. The intra-operative and post-operative course for this patient was unremarkable and there was no history of any pre-existing medical conditions that would have predicted the CVA. The second patient was 76 years old with a history of heart disease. This patient expired on postoperative day 16 due to complications of urosepsis. 


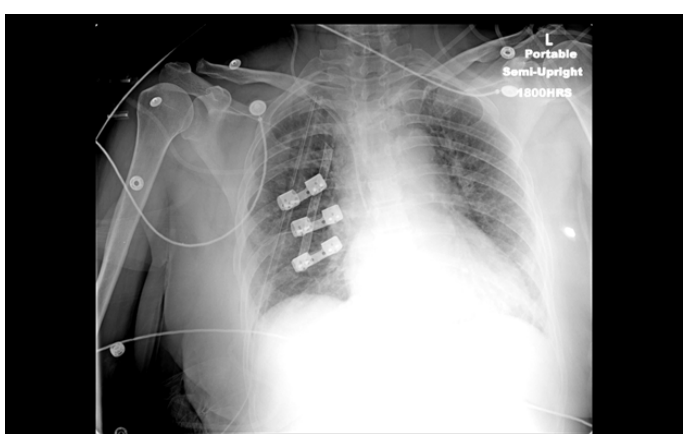

Figure 1: Post-operative chest radiograph demonstrating right sided rib fracture plating in patient

The intra-operative and post-operative course for this patient was uneventful following the plating of 2 displaced fractures due to severe pain despite morphine PCA. On POD 7, this patient developed sepsis and evaluation identified the genitourinary tract as the source of infection. There were no clinical indicators to associate the fracture plates as a source of infection in this patient. Despite adequate therapy, the patient continued to have a worsening course and on POD 16, withdrawal of care was implemented at the request of the family. Interestingly, both of these patients had calculated TRISS scores that had predicted favorable probability of survival, (TRISS scores 94.3 and 92.9 respectively). This underscores one of the limitations of the TRISS scale, in that it does not take into account co-existing medical conditions that may impact eventual outcome.

The median length of hospital stay for the cohort following surgery was 8 days with a range between 2 and 16 days. Additional procedures included orthopedic treatment of two patients with extremity fractures. Nine of the discharged 23 patients (39\%) required a skilled nursing facility for additional rehabilitation. Pain management strategy following surgery included implementation of oral narcotics and non-steroidal anti-inflammatory (NSAID) agents immediately following surgery and the removal of pre-existing epidural catheters or intravenous narcotics within 48 hours of surgery. This period coincided with removal of existing pleural drains. Three patients required mechanical ventilation greater than 48 hours and in these patients, the pleural drains were left in situ until liberation from the ventilator. All patients were discharged on oral narcotic medications and non-steroidal anti-inflammatory agents for one month.

\section{Level of pain and patient satisfaction}

As a group, the cohort represents a subgroup of BTT patients who had rib injury and severe pain. The average pain intensity was recorded as greater than or equal to 7 (severe pain) in all 25 patients prior to fracture stabilization. For the entire cohort, the mean NPI score prior was $8.9 \pm 1.1$. In fact, thirteen patients (52\%) were experiencing maximum pain with a NPI score of 10 despite analgesic therapy. Following rib stabilization, there was a dramatic reduction in the level of pain among the cohort of patients (Figure 2). Twenty two of the 25 patients (88\%) had a decline in their level of pain (NPI) within 48 hours of rib fracture reduction and stabilization. The observed mean NPI scores at 24 and 48 hours following rib fracture treatment were 4.7 \pm 1.7 and $3.3 \pm 1.2$ respectively $(\mathrm{p}<0.05)$. At hospital dismissal, the level of experienced pain continued to decline further to $2.9 \pm 1.3(\mathrm{p}<0.05)$ and at late follow up, the NPI score was $1.7 \pm 1.5(\mathrm{p}<0.05)$. Patient satisfaction, as assessed by willingness to undergo the procedure again was positive in the 22 patients (88\%). The two patients who expired were both among the group with a positive response following the procedure. Three patients said they would not undergo the procedure again. All 3 of these patients experienced less pain after plating; however, all three expected a greater relief of discomfort than actually observed.

\section{Follow-up}

Follow up information was available in 21 of $25(84 \%)$ patients at a median of 4 months (range 1 to 24 months) after hospital dismissal. Two of the patients had expired, with the cause of death being unknown in both patients. The first patient was a 60 year old female who had fallen and sustained isolated rib fractures. This patient had noted significant improvement in her discomfort following rib plating. The second patient was a 48 year old female, who also had fallen and experienced only rib injury; however, this patient was one of three patients noted above who were dissatisfied with the results of surgery. Of the 19 patients seen in follow up clinic, no one had any late wound complications related to the fracture plates. With the exception of three patients who had pre-existing chronic pain issues necessitating maintenance narcotics, none of the 16 other patients at follow up were requiring pain medications. All of these 19 patients had returned to their previous level of activity without physical limitations related to rib fracture pain or the surgical incision required to repair.

\section{Discussion}

Chest wall trauma can be a significant contributor to morbidity and mortality in the injured patient [1,2]. Current management strategy of such patients is largely defined by the presence or absence of flail chest wall deformity and concomitant organ dysfunction [3-5]. Among patients with lesser extent of trauma, such as those sustaining isolated rib fractures, the goal of management is upon sufficient analgesia [3-5]. However, a group of patients will continue to have severe pain (NPI >7) despite all pharmacological pain-relieving maneuvers and pose a formidable challenge to the clinician [7-9]. Unresolved chest wall pain can have significant negative consequences, such as ineffectual cough, retained secretions, atelectasis, pneumonia and respiratory failure [1]. Established upon the findings of this writing, rib fracture reduction and stabilization, utilizing the less invasive deployment systems available, appears to be effective pain control when other modalities fail. This conclusion is supported by the observation that significant and sustained pain alleviation was noted among the study group as noted by the significant NPI score reduction (Figure 2). The group as a whole exemplifies a difficult pain control situation. As an illustration of the severity, the initial treatment of this cohort included $36 \%$ with epidural analgesia and 21 of 25 patients (84\%) with either neuraxial or intravenous analgesia (PCA) in place with continued suboptimal pain control. In fact, over half (52\%) of the patients who underwent the procedure recorded their level of pain at 10 (most severe) just prior to the procedure. Remarkably, almost all of the patients studied (88\%) had a significant reduction in pain following fracture plating. This dramatic improvement in the perceived level of pain was noted, despite the requirement of access incisions for the positioning of the rib fracture plates. Overall patient satisfaction with the procedure correlated with the reduction in pain and was observed in the majority of patients $(88 \%)$, almost all of whom stated they would have the procedure again.

Concerns regarding additive morbidity or mortality to injured patients were not observed in this study as there were no detected perioperative complications related specifically to rib plating. Of the 


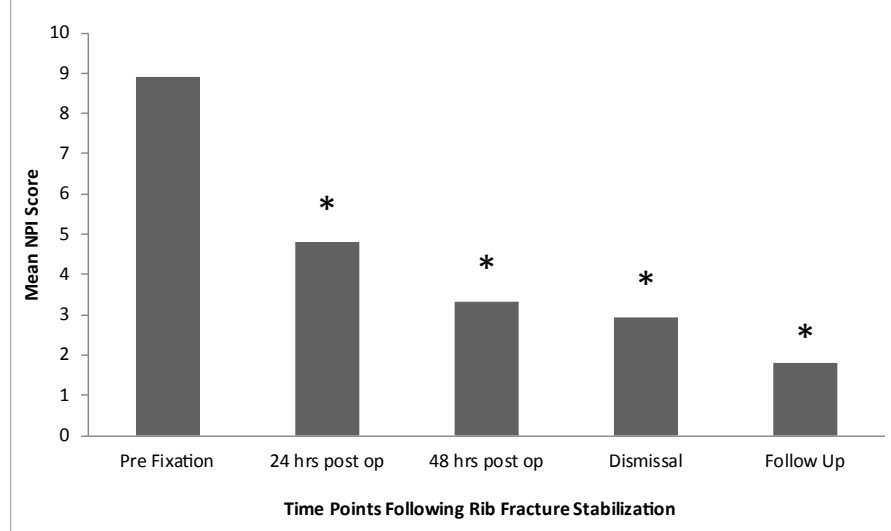

Figure 2: Mean Numeric Pain Intensity scores prior to and following open reduction and internal fixation of displaced rib fractures at various time points. ${ }^{*} p<0.05$.

two patients who expired while in hospital, both died due to conditions unrelated to the incipient trauma or the plating procedure. The decision to perform such a procedure in an elderly patient should be balanced between the beneficial results of less narcotic requirements and improved respiratory mechanics with the morbidity of a surgical procedure. Although the observed mortality is not directly related to the rib plating procedure, the deaths of these two patients emphasize the importance of a physician group discussion of all participating specialists prior to embarking upon this form of therapy.

At longer follow up, there were no observed wound complications or late consequences from the fracture plates left in situ. Most of the patients who followed up were back to their regular activities of daily living. It should be noted however, that not all patients were satisfied and highlights the importance of appropriate patient selection. The 2 patients who noted suboptimal results were patients on chronic pain management regimens. It remains unclear if rib plating should be offered to such patients, as they are often, the most challenging patients to achieve pain control. Conceivably, if rib plaiting can improve the perception of pain, then one could hypothesize that such an intervention will aid in the management of such complicated situations. Another question that remains is whether rib fracture plating can benefit patients with non-displaced rib fractures. Although these patients experience substantial agony, this study was limited to those with displaced fractures as these patients were expected to experience the most severe degrees of pain. Further analysis of patients without displacement will be useful and may expand the application of rib plating technology to more trauma patients.

Limitations of this paper are that this is a retrospective analysis of a small cohort of patients from a single institution. Without the benefit of a matched comparison group, the findings of this study can be scrutinized regarding statistical validity. However, the dramatic findings of this paper regarding the finding of pain relief as noted consistently in the collective group of patients lends credibility to the conclusions cited, despite statistical shortcomings.

It is this author's opinion that rib fracture plating should not supplant established pain management strategies for thoracic trauma patients. The purpose of this paper was to determine if rib fracture reduction and stabilization could control pain in a select group of patients as outlined above. This form of therapy should be considered adjunctive to recommend pain pathways and not stand alone therapy.

\section{Conclusion}

Rib fracture open reduction and internal plating fixation should be considered in the pain management algorithm of lesser injured BTT patients who are experiencing severe pain due to displaced rib fractures.

\section{References}

1. Cogbill TH, Landercasper J (2000) Injury to the chest wall. (4thedn), New York, NY: McGraw-Hill.

2. Ziegler DW, Agarwal NN (1994) The morbidity and mortality of rib fractures. $J$ Trauma 37: 975-979.

3. Simon BJ, Cushman J, Barraco R, Lane V, Luchette FA, et al. (2005) Pain management guidelines for blunt thoracic trauma. J Trauma 59: 1256-1267.

4. (2006) EAST Practice Management Guideline for the treatment of Pulmonary Contusion - Flail Chest.

5. Pettiford BL, Luketich JD, Landreneau RJ (2007) The management of flail chest. Thorac Surg Clin 17: 25-33.

6. de Moya M, Bramos T, Agarwal S, Fikry K, Janjua S, et al. (2011) Pain as an indication for rib fixation: a bi-institutional pilot study. J Trauma 71: 1750-1754.

7. Kerr-Valentic MA, Arthur M, Mullins RJ, Pearson TE, Mayberry JC (2003) Rib fracture pain and disability: can we do better? J Trauma 54: 1058-1063.

8. Gasparri MG, Tisol WB, Haasler GB (2010) Rib stabilization: lessons learned Eur J Trauma Emerg Surg 36: 435-440.

9. Karmakar MK, Ho AM (2003) Acute pain management of patients with multiple fractured ribs. J Trauma 54: 615-625. 